\title{
Changes in design driving load cases: Operating an upwind turbine with a downwind rotor configuration
}

\author{
Wanke, Gesine; Bergami, Leonardo; Larsen, Torben J.; Hansen, Morten Hartvig
}

Published in:
Wind Energy

Link to article, DOI:

10.1002/we.2384

Publication date:

2019

Document Version

Peer reviewed version

Link back to DTU Orbit

Citation (APA):

Wanke, G., Bergami, L., Larsen, T. J., \& Hansen, M. H. (2019). Changes in design driving load cases: Operating an upwind turbine with a downwind rotor configuration. Wind Energy, 22(11), 1500-15811.

https://doi.org/10.1002/we.2384

\section{General rights}

Copyright and moral rights for the publications made accessible in the public portal are retained by the authors and/or other copyright owners and it is a condition of accessing publications that users recognise and abide by the legal requirements associated with these rights.

- Users may download and print one copy of any publication from the public portal for the purpose of private study or research.

- You may not further distribute the material or use it for any profit-making activity or commercial gain

- You may freely distribute the URL identifying the publication in the public portal 


\section{SDUণ́ \\ University of Southern Denmark}

\section{Changes in design driving load cases}

operating an upwind turbine with a downwind rotor configuration

Wanke, Gesine; Bergami, Leonardo; Larsen, Torben J.; Hansen, Morten H.

\section{Published in:}

Wind Energy

DOI:

10.1002/we.2384

Publication date:

2019

Document version

Accepted manuscript

Citation for pulished version (APA):

Wanke, G., Bergami, L., Larsen, T. J., \& Hansen, M. H. (2019). Changes in design driving load cases: operating an upwind turbine with a downwind rotor configuration. Wind Energy, 22(11), 1500-1511.

https://doi.org/10.1002/we.2384

\section{Terms of use}

This work is brought to you by the University of Southern Denmark through the SDU Research Portal.

Unless otherwise specified it has been shared according to the terms for self-archiving.

If no other license is stated, these terms apply:

- You may download this work for personal use only.

- You may not further distribute the material or use it for any profit-making activity or commercial gain

- You may freely distribute the URL identifying this open access version

If you believe that this document breaches copyright please contact us providing details and we will investigate your claim.

Please direct all enquiries to puresupport@bib.sdu.dk 


\section{Changes in design driving load cases; operating an upwind turbine with a downwind rotor configuration.}

\section{Gesine Wanke*1 | Leonardo Bergami ${ }^{1}$ | Torben J. Larsen ${ }^{2}$ | Morten H. Hansen ${ }^{3}$}

${ }^{1}$ Blade Science Center, Suzlon, Vejle, Denmark

${ }^{2}$ DTU Wind Energy, Technical University of Denmark, Roskilde, Denmark

${ }^{3}$ Mads Clausen Institute, University of Southern Denmark, Sønderborg, Denmark

\section{Correspondence}

*Gesine Wanke, Suzlon Blade Science Center, Havneparken 1, 7100 Vejle, Denmark. Email: gesine.wanke@suzlon.com

Present Address

Suzlon Blade Science Center, Havneparken 1, 7100 Vejle, Denmark.

\begin{abstract}
Summary
This work considers the design driving load cases from a full design load basis analysis on an upwind turbine changed into a downwind configuration. The upwind turbine is a commercial class IIIA 2.1MW turbine, manufactured by Suzlon. The downwind turbine shows an increase in the normalized tower clearance by $6 \%$, compared to the upwind concept. Removing the blade prebend increases the normalized minimum tower clearance by $17 \%$ in the downwind configuration compared to the upwind configuration. The extreme loads on the longitudinal tower bottom bending moment are seen to generally increase by $17 \%$ due to the overhanging gravity moment of the rotor-nacelle assembly. The extreme blade root bending moments are reduced by $10 \%$ flapwise, due to the coning of the rotor in downwind direction. The fatigue loads suffer from the tower shadow, leading to an overall increase of the fatigue loads in the blades with up to $5 \%$ in flapwise direction in the downwind configuration. Due to blade deflection and coning direction, the downwind configuration shows a $0.75 \%$ lower annual energy production. Removing the prebend increases the annual energy production loss to $1.66 \%$.
\end{abstract}

KEYWORDS:

downwind rotor, design loads basis, extreme loads, fatigue loads

\section{1 | INTRODUCTION}

Over the recent years, most research effort has been focused on wind turbines with an upwind concept, where the rotor is placed in front of the tower with respect to the wind direction. The aeroelastic behavior of wind turbines is well known for upwind concepts. In this case, the clearance between tower and blades is a major restriction in the blade design. Placing the rotor behind the tower could give the freedom of larger rotors for higher power production or more flexible blades, saving on the blade material and potentially decreasing the loads on tower, nacelle and hub. Although, load cases of fault situations, such as the emergency stop of the turbine, where the blades are unloaded, may restrict the blade flexibility with a minimum tower clearance in a downwind configuration.

This work investigates the following hypothesis: changing an upwind into a downwind concept leads to major changes in the design driving load cases, increasing the freedom for blade design.

According to Kiyoki ${ }^{1}$ only the Japanese manufacturer Hitachi is commercially selling a downwind concept of $2 \mathrm{MW}$ and testing a $5 \mathrm{MW}$ prototype. The relaxation of the tower clearance in the design constraints made the concept attractive for typhoon sites.

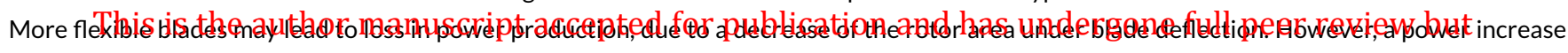

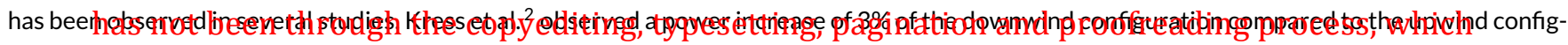
uration ina mamputational Flyid Bynamic (CFD) simulations. The authors gbserved a higher output due to a pigher angle of attack across the blade spanwise direction originating from a favorable blockage effect of the nacelle in the downwind configuration. Yoshida et al. 3 observed an increase in annual energy production (AEP) by $7.5 \%$ in field tests, comparing an upwind configuration against a downwind configuration in complex terrain. 
The study showed a power increase due to the rotor alignment with the inclination angle of the wind field of 1 to $11 \%$. In the downwind configuration, the tilt angle counteracted the inclination angle of the wind resulting into a more perpendicular inflow on the rotor plane, than in the upwind configuration.

Ning and Petch ${ }^{4}$ compared the cost advantage of upwind concepts of $5-7 \mathrm{MW}$ turbines for different wind classes with corresponding downwind concepts. In their work, a multidisciplinary optimization framework was used to design several rotors of different diameter for different wind conditions, with and without tower clearance constraint. Their work showed clear dependencies of the results on the site conditions. The highest cost advantage of a downwind concept was found for the $5 \mathrm{MW}$ turbine for sites of lower average wind speed and moderate turbulence, with a cost advantage of 1-2\% of levelized cost of energy (LCOE). Sites with higher average wind speeds showed an increase in cost, due to the increase in tower bending moments from extreme loads, caused by the overhanging moment of the rotor. Furthermore, a clear dependency of the LCOE on the rotor diameter was observed, showing the lowest LCOE for medium sized rotors.

The downwind concept comes further with the option of a passive yaw mechanism for the alignment of the rotor, as the aerodynamic forces could potentially align the rotor with the wind direction. It should be noted that the advantage of a passive yaw mechanism comes from the elimination of load cases with extreme yaw error in the design load assessment. These cases are often associated with very high loads that are restricting the turbine design. The cost advantage of a passive yaw system in itself is small, since a yaw mechanism will still be required for cable unwinding. Glasgow and Corrigan ${ }^{5}$ investigated the alignment and stability of a passive yaw system already in 1983 , by testing the passive yaw alignment of different rotors in a downwind configuration on a $100 \mathrm{~kW}$ machine. They observed significant differences in the equilibrium yaw position, where some rotors were not able to align passively with the wind direction. Also they observed yaw motions around these equilibrium positions. If the equilibrium position of the rotor shows severe misalignment with the wind direction, power losses can be expected, as shown in field measurements already by Corrigan and Viterna ${ }^{6}$ in 1982.

The major challenge of a downwind concept is the change of loading on the blades when they are passing the tower. The velocity deficit behind the tower gives a reduction in normal force on the blade of about $20 \%$, as observed for example by Zahle et al. 7 The velocity deficit leads also to a rapid fluctuation in the angle of attack as the blade passes through the wake.

Glasgow et al. ${ }^{8}$ compared the flapwise bending loads of a blade of a $100 \mathrm{~kW}$ machine with lattice tower in an upwind configuration and a downwind configuration. While they were not able to measure a difference in the mean flapwise bending load, they observed a significant increase in the cyclic flapwise bending load within one rotor revolution due to the flow disturbance from the tower.

Reiso and Muskulus ${ }^{9}$ studied the fatigue load of a downwind configuration compared to an upwind configuration of the $5 \mathrm{MW}$ NREL reference turbine. They observed an increase of around $20 \%$ in the damage equivalent fatigue load for the flapwise blade root bending moment and for the tower base bending moment. Their study further investigates the reduction of the fatigue load due to an airfoil-shaped tower fairing combined with a mass and stiffness reduction of the blade. This approach shows $5 \%$ fatigue load reduction for the blade root flapwise bending moment compared to the upwind configuration. However, due to the size of the fairing the rotor overhang had to be increased. This resulted into a mean tower bottom bending moment increase by around $20 \%$.

For this numeric study, a commercial 2.1MW Suzlon turbine is changed from an upwind configuration into a downwind configuration by realigning the turbine components. Assembling the turbine with the same components could be economically beneficial, as blade moulds could be reused. As the direct change of configuration is seen to be only little beneficial a downwind configuration without blade prebend is also compared to the upwind configuration. Full design load basis are calculated according to the IEC-Standard 10 and the three configurations are compared in terms of minimum tower clearance, the extreme loads and the fatigue loads. From the load basis calculation the design driving load cases are identified. The 10-minute mean load is shown for certain sensors to explain major load differences between the upwind and the downwind configurations. It is shown that the design driving load situations for the tower clearance change from high blade loading in the upwind configuration to negative blade loading in the downwind configurations. The flapwise blade root moment is seen to decrease due to the coning direction in the downwind configurations when compared to the upwind configuration. The edgewise blade root moments for the downwind configurations are observed to increase for high wind speeds, due to the projection of the centrifugal force. It is confirmed that the tower loads increase in the downwind configurations relative to the upwind configuration due to the overhanging moment of the rotor nacelle assembly. While the flapwise fatigue load of the blade root is seen to increase in the downwind configurations, compared to the upwind configuration, purely due to the tower shadow, the edgewise blade root fatigue load is further increasing due to lower damping of the edgewise forward whirling turbine mode. AEP losses for the downwind configurations due to the tower shadow and the effect of prebend on the AEP are quantified. Finally, the fluctuation of the produced power is assessed.

\section{2 | METHODS}

For the study the Suzlon 2.1MW turbine S111 (wind class IIIA) is used. The turbine has a rotor diameter of $112 \mathrm{~m}$ and a tubular tower of $90 \mathrm{~m}$ height. The rotor is tilted and coned. The blades are prebended. The turbine is pitch regulated at a rated wind speed of $9.5 \mathrm{~m} / \mathrm{s}$ and the operational range is 


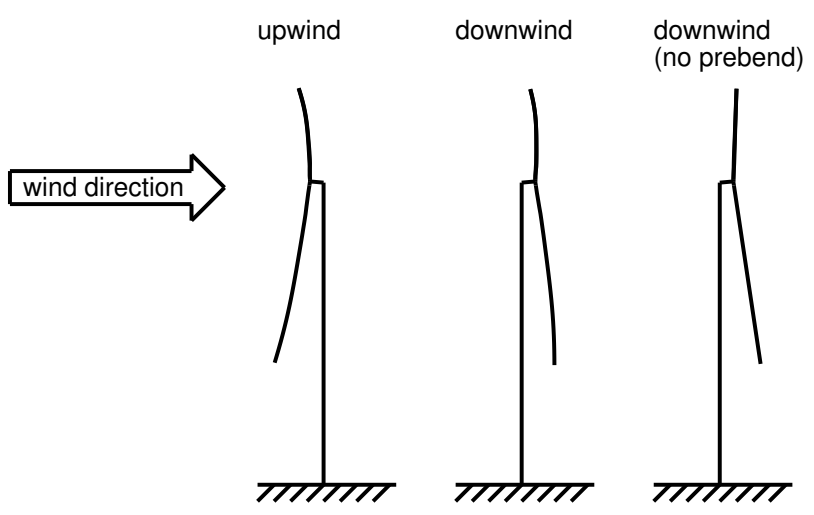

FIGURE 1 Sketch of the side view of the the turbine configurations upwind, downwind and downwind without prebend.

simulated between $4 \mathrm{~m} / \mathrm{s}$ and $21 \mathrm{~m} / \mathrm{s}$.

To compare the upwind configuration with the downwind configuration, the downwind configuration is reassembled with the same turbine bodies: The rotor is moved behind the tower, the nacelle structure with the shaft is rotated $180^{\circ}$ around the yaw axis. The assembly results into a downwind configuration, where tilt and cone increase the clearance between blades and tower. The rotational direction seen from a global frame of reference is clockwise for all configurations when looking at the turbine with the wind in the back. Since the blades are prebended towards the pressure side, the prebend increases the distance between blades and tower in the upwind configuration, but decreases it in the downwind configuration. The unloaded tower clearance, normalized with the distance between the blade tip and tower of the upwind configuration, is $100 \%$ for the upwind configuration, while the downwind configuration has an unloaded tower clearance of only $62 \%$. In a more realistic design, the downwind rotor blade has no prebend, to increase the freedom in the blade design. The downwind configuration without prebend is therefore also simulated to see the effect of the prebend. The downwind configuration without prebend has an unloaded tower clearance of $81 \%$. Figure 1 phows a sketch of the three evaluated configurations from the side view, scaled from the original turbine measures without any loading.

A full design load basis (DLB) is calculated with HAWC2 ${ }^{11}$ simulations (Version 12.7), according to IEC $61400-1$ standard edition 3 10. The IEC 61400-1 standard gives a guideline to calculate loads on the wind turbine created within different situations (Design Load Cases (DLC)). These situations regard normal operation with different wind conditions (DLC 1.x), failure of the control system or the electrical systems (DLC 2.x), startup and shut-down scenarios (DLC 3.x to DLC 5.x), scenarios with parked turbine configurations (DLC 6.x) and situations with a locked rotor (DLC 7.x and DLC 8.x). The standard prescribes the turbulence intensity for each load case and wind speed, as well as the wind shear to be applied. To each design load case, a different safety factor is assigned by the standard. For the load calculation the DLB is set up according to the design load basis for onshore turbines by Hansen et al. ${ }^{12}$, which is a fully documented interpretation of the IEC standard. The design load basis contains a tabulated description of the wind field parameter and safety factors. The simulation setup applies the Beddoes-Leishman dynamic stall model and Prandtl's tipp loss correction for the aerodynamics of the blade. Yawed inflow is regarded by HAWC2 by adjusting the mean induction and applying a correction on the induction depending on the azimuth position of the blade. The background of the model has been described by Madsen et al. 13 . All simulations are performed with a pitch imbalance of $\pm 0.3^{\circ}$ and a mass imbalance of $1 \%$ on one rotor blade. All simulations are performed with a flow inclination angle of $8^{\circ}$. It is assumed that the turbine operates under normal condition $50 \%$ of the time at $0^{\circ}$ average yaw angle and $25 \%$ of the time at $+7^{\circ}$ and $-7^{\circ}$ average yaw angle. The number of turbulence seeds realized are according to the DLB and the same turbulence seeds are used for the upwind and the downwind configurations. For the upwind configuration, a potential flow tower shadow model is used, while a jet-flow deficit model is used for the downwind configurations, as described in the HAWC2 manual 11. For simulations with a wind direction of yaw errors between $+90^{\circ}$ and $+270^{\circ}$, the upwind configuration is subject to the jet-flow deficit model and the downwind configuration is subject to the potential flow model. An exception are rotating wind fields, where the range of wind direction covers $360^{\circ}$. Here the jet-flow deficit tower shadow model is applied for the downwind configurations and the potential flow model for the upwind configuration. Additionally, the downwind configurations have been simulated with a potential flow model during normal operation to quantify the effect of the tower shadow model, especially on fatigue loads and the AEP.

For "publishability", the controller set-up from the technical university of Denmark (DTU) 14 has been adopted. It is a controller for pitch regulated, variable speed wind turbines with a partial load and full load control strategy, as well as a switching routine between the two. In the partial load region, the balance between generator torque and aerodynamic torque is used for optimal $C_{p}$ tracking. In the full load region, torque limits are applied by following a constant torque or constant power strategy. Different events can be initiated from the controller, such as start-up, shut-down or failure situations. For the design load case $2.2 \mathrm{~b}$, failure of pitch systems (one blade getting stuck), the suggested routine from Hansen et al. has been changed. In the failure scenario the pitch actuator has been adjusted according to the controller routine of the turbine. In this case, the pitch 
angle on one blade will be kept constant at the current pitch angle at the time of failure. The deviation of the pitch angle from the set point of the controller initiates a stop of the turbine. Further, a pitch run away (DLC 2.2p) is not included, as the failure mode is prevented from the type of pitch actuators used. For the scenario of a parked turbine with high yaw errors (DLC 7.1), the yaw error is interpreted as wind direction change of $360^{\circ}$ within 570 seconds.

For the AEP and fatigue load calculation, a mean wind speed of $7.5 \mathrm{~m} / \mathrm{s}$ and a shape factor $\mathrm{k}=2$ are used for the Weibull distribution of the wind, as specified for class III turbines in the IEC standard. The power curves are calculated with wind speeds steps of $1.0 \mathrm{~m} / \mathrm{s}$ with 6 turbulence seeds per wind speed bin with the normal turbulence model and without yaw error. For further investigation on energy production the AEP is also calculated from simulations without inclination angle. The normalized power fluctuation is regarded for each wind speed bin to investigate the power quality. Therefore the standard deviation of the power is divided by the mean of the power for each wind speed bin. From the load simulations including the inclination angle the 10-minute mean load is calculated over wind speed to show general changes in loading between the configurations.

For the post-processing of the extreme values, the safety factors are applied and the resulting extreme values are averaged over the six highest extremes from simulations differing only in turbulence seeds. If less than six turbulence seeds were simulated, the average represents values from all the available seeds. For the tower clearance the safety factor is applied to the blade deflections. The normalized tower clearance $\mathrm{TC}_{\text {normalized }}$ is calculated from the simulated tower clearance $\mathrm{TC}_{\text {simulated }}$ as

$$
T C_{\text {normalized }}=\frac{T C_{\text {unloaded }}(1-S F)+T C_{\text {simulated }} S F}{T C_{\text {upwind }} \text {, unloaded }}
$$

Where $\mathrm{TC}_{\text {unloaded }}$ is the unloaded tower clearance individual for each configuration, $\mathrm{TC}_{\text {upwind, unloaded }}$ is the unloaded tower clearance of the upwind configuration and SF is the combined safety factor containing a load case specific contribution and a contribution for the elastic properties of the material. The tower clearance is calculated as an average over simulations with different turbulence seeds.

Further investigations had to be made regarding fatigue loads and the stability tool HAWCStab2 ${ }^{15}$ (Version 2.14) has been used to determine the damping of certain aeroelastic turbine modes.

\section{3 | RESULTS}

In the following section, the results of the load simulations for the full design load basis are presented. Presented are the minimum tower clearance, extreme loads and fatigue loads for selected sensors and the power production. For the minimum tower clearance and the extreme values the three most extreme results are plotted to give a better overview about the general load level. However, design driving for the load sensor is only the design load case giving the highest extreme load or the minimum tower clearance. The selected load sensors are the blade root moment sensor, the main bearing yaw and tilt sensor in a non rotating reference frame, and the tower bottom sensors. These sensors are selected as they represent the loads on the most costly components of the turbine. These sensors also give a good overview over the over all trends in change in loading situations when changing the upwind configuration into a downwind configuration. However, with only being able to present a limited number of sensors this work does not present a complete picture of all load sensor on a wind turbine. All results are shown as relative comparison between the downwind configurations and the upwind configuration. They are expressed relative to the result of the upwind configuration. The results for the different load cases are identified with the number of the design load case (DLC), the wind speed (ws) and the wind direction (dir). Blade root bending moments in flap and edgewise are stated in the pitched blade root coordinate system.

\section{1 | Tower clearance}

The tower clearance is normalized with the distance of the unloaded blade tip to the tower of the upwind configuration. Figure 2 hows the comparison of the normalized tower clearance for the upwind, the downwind and the downwind configuration without prebend.

The figure shows, that none of the configurations is subject to tower strikes. Under loading the tower clearance has decreased to $6 \%$ in the upwind configuration, to $12 \%$ in the downwind configuration and to $23 \%$ in the downwind configuration without prebend. The deflection of the blade tip in the upwind configuration from the unloaded position towards the tower is $94 \%$, due to the loading. In the downwind configuration the blade tip deflects from an unloaded position of $62 \%$ by $50 \%$ under loading. The downwind configuration without prebend shows under loading a blade tip deflection of $58 \%$ decreasing tower clearance from $81 \%$ to $23 \%$. With the change of configuration the load cases driving the minimum tower clearance have not changed. Determining is the operation in extreme turbulence (DLC 1.3). The critical wind speeds have changed from wind speeds around rated power in the upwind configuration, to high wind speeds in both downwind configurations.

Characteristic cases in the upwind configuration regarding the minimum tower clearance are cases with high blade loading. The high loading leads to high deformations, for example the operation at extreme turbulence around the thrust peak. In contrast to this, the characteristic situations for the minimum tower clearance in the two downwind configurations are situations, where the blades experience a negative lift force due to the 


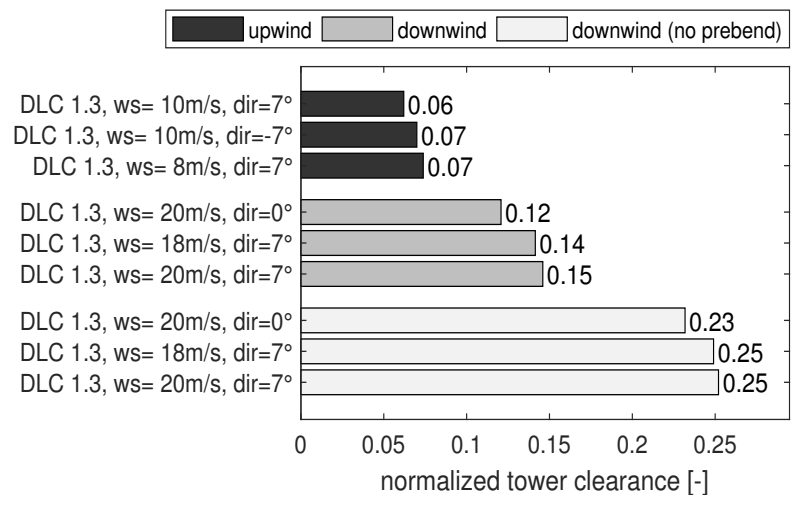

FIGURE 2 Normalized tower clearance compared between the upwind, the downwind and the downwind configuration without prebend from the load cases with the three lowest values. Design load case: DLC 1.3: power production in extreme turbulence.
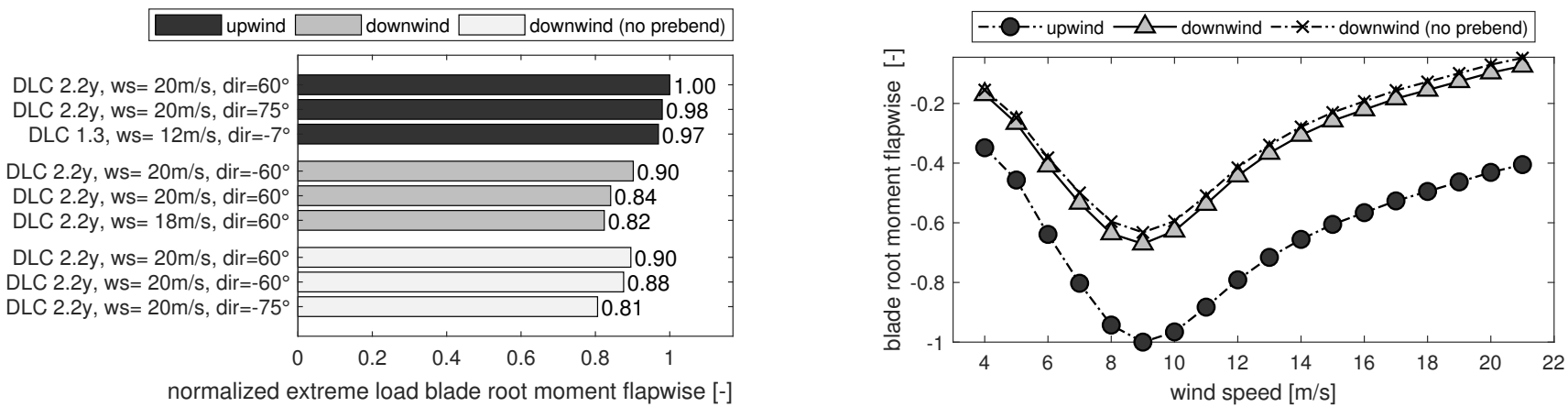

FIGURE 3 Comparison of flapwise blade root bending moments between the upwind, downwind and the downwind configuration without prebend. Left: comparison of the highest three normalized flapwise blade root bending moments. Right: comparison of the normalized 10-minute mean flapwise blade root bending moments over wind speed. Design load cases are: DLC 2.2y power production with abnormal yaw error and DLC 1.3 power production in extreme turbulence.

operation at high wind speeds and high pitch angles. Due to this change in characteristic situation, the lower blade deflection in the two downwind configurations can overcome the lower unloaded tower clearance.

\section{2 | Extreme loads}

In the following subsection, the extreme load results are shown. The extreme loads are normalized with the highest load observed for the upwind configuration. For further explanation of the results, the 10-minute mean load is shown over wind speed for selected sensors.

Figure 3 hows the normalized extreme and the normalized 10-minute mean flapwise blade root bending moments for the upwind configuration, the downwind configuration and the downwind configuration without prebend.

The figure shows on the left that the extreme flapwise blade root bending moment is lower in both downwind configurations compared to the upwind configuration. The extreme blade root bending moment reduces $10 \%$ when the upwind configuration is changed into a downwind configuration. The design driving load cases are determined by operation at extreme yaw error (DLC 2.2y) at high wind speeds. The analysis of the 10-minute mean flapwise bending moment shows on the right of Figure 3 that the mean flapwise blade root bending moment at the thrust peak is $33 \%$ lower in the downwind configuration than in the upwind configuration. The downwind configuration without prebend shows a mean load reduction of $37 \%$ at the thrust peak compared to the upwind configuration for the flapwise blade root bending moment.

When the blades are loaded in the upwind configuration the blade tip deflection will increase the rotor area, counter acting the prebend and the cone angle. As the downwind configurations are coned in the same direction as the blade tip deflects under loading, any deflection further out of the rotor plane than the straight blade will decrease the rotor area and unload the rotor blades. The effect of the change of cone direction, when the upwind configuration is changed into the downwind configuration, can be seen from the mean flapwise bending moments to have the 




normalized extreme load blade root moment edgewise [-]



FIGURE 4 Comparison of edgewise blade root bending moments between the upwind, downwind and the downwind configuration without prebend. Left: comparison of the highest three normalized edgewise blade root bending moments. Right: comparison of the normalized 10-minute mean edgewise blade root bending moments over wind speed. Design load case is: DLC 1.3 power production in extreme turbulence.

highest load reducing effect. The increased out of plane deflection from the downwind configuration without prebend has a small load decreasing effect on the mean flapwise bending moments compared to the effect of cone. The maximum flapwise blade root bending moments are in both downwind configurations the same. From the time series of the according simulations, it is observed that the blade deflection of the two downwind configurations is very similar, in cases of high yaw errors. Therefore, there is no unloading effect due to larger out of plane blade deflection in DLC $2.2 \mathrm{y}$, when the prebend is removed from the downwind configuration.

Figure 4 hows the normalized extreme and 10-minute mean loads of the edgewise blade root bending moments for the upwind configuration, the downwind configuration and the downwind configuration without prebend.

The figure shows a 5\% higher extreme edgewise blade root bending moment in the downwind configuration compared to the upwind configuration. The downwind configuration without prebend shows a $11 \%$ higher extreme edgewise blade root bending moment than the upwind configuration. Design driving is for all configurations the operation at extreme turbulence for wind speeds close to the thrust peak at yaw errors. For the downwind configuration a high edgewise blade root bending moment is also observed for high wind speeds. From the 10-minute mean of the edgewise bending moment, a change in the load distribution over wind speed is observed, when the upwind configuration is changed into a downwind configuration. In the upwind configuration the mean blade root edgewise bending moment increases with the wind speed, and decreases strictly after reaching a maximum at $8 \mathrm{~m} / \mathrm{s}$. In the downwind configurations the mean edgewise blade root bending moments increase with the wind speed until the thrust peak at $9 \mathrm{~m} / \mathrm{s}$. However, after a decrease in the edgewise blade root bending moment for wind speeds up to $14 \mathrm{~m} / \mathrm{s}$, an increase in the mean edgewise blade root bending moment is observed for high wind speeds.

The difference in the mean edgewise blade root moment is mainly due to the projection of the centrifugal force. The combination of cone and pitch angle lead to a contribution of the centrifugal force to the mean edgewise bending moment (with $\sin ($ pitch) $\sin ($ cone)). For the operational point $17 \mathrm{~m} / \mathrm{s}$ the centrifugal force contributes to the mean edgewise bending moment with $-2.5 \%$ in the upwind configuration and with $+2.5 \%$ to the downwind configuration. $2.5 \%$ of centrifugal load are equivalent to $32 \%$ of mean edgewise blade root bending moment of the upwind configuration at the thrust peak. The change of the sign from the contribution of the centrifugal load, is due to the inverted sign of the cone angle when the upwind configuration is changed into the downwind configuration. Thus, the difference observed in the edgewise blade root moment mean load is due to the projection of the centrifugal force to the edgewise bending moment. Further investigations of the aeroelastic modes with HAWCStab2 showed that the first edgewise forward whirling mode is significantly lower damped around rated power in the downwind configuration. Removing the prebend decreases the damping in the downwind configuration even further. For high wind speeds, close to cut-out wind speed, the damping increases for both downwind configurations. Due to this change in damping the load fluctuations around rated power are higher than for the upwind configuration. Therefore a significant increase in extreme loads can be observed for the edgewise blade root bending moment and the downwind configuration without prebend shows the highest extreme load level. Whether a lower damped first edgewise forward whirling mode is general for downwind configurations and which parameters are influencing it, needs to be investigated in future work.

Figure 5 hows the comparison of the the tower bottom bending moment in longitudinal direction for the upwind configuration, the downwind configuration and the downwind configuration without prebend. The normalized extreme load is displayed on the left and the normalized 10-minute mean load over wind speed on the right.

It can be seen that the extreme load level for the longitudinal tower bottom moment is generally higher in the two downwind configurations than in the upwind configuration. The highest extremes of the longitudinal tower bottom moment are a result from the shut-down during extreme 




normalized extreme load tower bottom moment longitudinal $[-]$

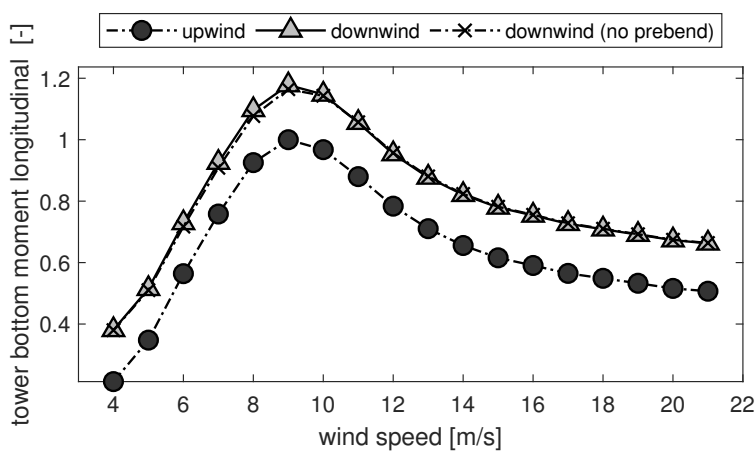

FIGURE 5 Comparison of longitudinal tower bottom bending moments between the upwind, downwind and the downwind configuration without prebend. Left: comparison of the highest three normalized longitudinal tower bottom bending moments. Right: comparison of the normalized 10minute mean longitudinal tower bottom bending moments over wind speed. Design driving load cases are: DLC 4.2 shut down during extreme operational gust, DLC 2.2y operation at extreme yaw errors, DLC 3.1 start up.

operational gust (DLC 4.2) and operation at extreme yaw errors (DLC 2.2y) for the upwind configuration. In the two downwind configurations, the highest extremes of the longitudinal tower bottom moment are determined by the shut-down at extreme operational gust and the start-up situation (DLC 3.1). From the 10-minute mean load, it is observed that the two downwind configurations are subject to higher longitudinal tower bottom bending moments. The mean longitudinal tower bottom bending moment at the thrust peak is $17 \%$ higher in the two downwind configurations than in the upwind configuration. There is no significant difference between the two downwind configurations.

Generally, start-up and shut-down situations can cause the longitudinal motion of the turbine, associated with high tower loads. The longitudinal tower bottom bending moment increases, due to the overhanging moment of gravity of the rotor-nacelle assembly in the two downwind configurations, compared to the upwind configuration. While the overhanging moment is counteracting the moment from the thrust force in the upwind configuration, the overhanging moment and the thrust force moment are acting in the same direction in the two downwind configurations. The increase in tower bottom bending moment in the downwind configurations due to this geometrical alignment of the gravity force is $17 \%$ compared to the maximum 10-minute mean load from DLC 1.2 in the upwind configuration. The overhanging moment is seen to be determining the load increase in the tower bottom bending moment. The difference in the overhanging moment of the rotor-nacelle assembly between the two downwind configurations is very small, since the contribution of the mass at the blade tip is small compared to the mass of nacelle, shaft and the blade root part. Therefore, the downwind configuration without prebend shows no significant difference in the the extreme loads.

Table 1 shows a summary of the design driving loads and load cases for the tower, blades and main bearing.

With the change from the upwind to the downwind configurations, the design driving tower bending loads (longitudinal and lateral), as well as edgewise blade bending and the torsional blade load are increasing. Also for the main bearing tilt load, a load increase is observed in the downwind configurations compared to the upwind configuration. The flapwise blade bending load, the tower torsional and the main bearing yaw load are lower in the downwind configurations than in the upwind configuration. The downwind configuration without prebend showed generally very similar load levels to the load level of the downwind configuration, differing maximum up to $3 \%$ from the downwind configuration. It can be seen that the design driving load cases are changing only in the main bearing tilt load with the change from an upwind to a downwind configuration. The design driving load cases are observed to be the same for both downwind configurations.

It should be mentioned that the absolute tower lateral bending moment of both downwind configurations is lower than the maximum tower longitudinal bending moment in the upwind configuration. Like the tower longitudinal bending moment, the main bearing tilt moment increases with the increased overhanging moment of the rotor-nacelle assembly in the downwind configurations compared to the upwind configuration. The tower torsional moment, as well as the bearing yaw moment, are related to the flapwise load of the blades and a load decrease in these sensors is observed, due to the coning in the direction of blade deflection in the downwind configurations.

\section{3 | Fatigue loads}

In the following section, the changes in fatigue loads due to the change in turbine configuration are discussed. Figure 6 shows the normalized lifetime equivalent load for the flapwise blade root bending moment, over the different load cases of the fatigue load assessment. The load is normalized with the total lifetime equivalent load from the upwind configuration. 


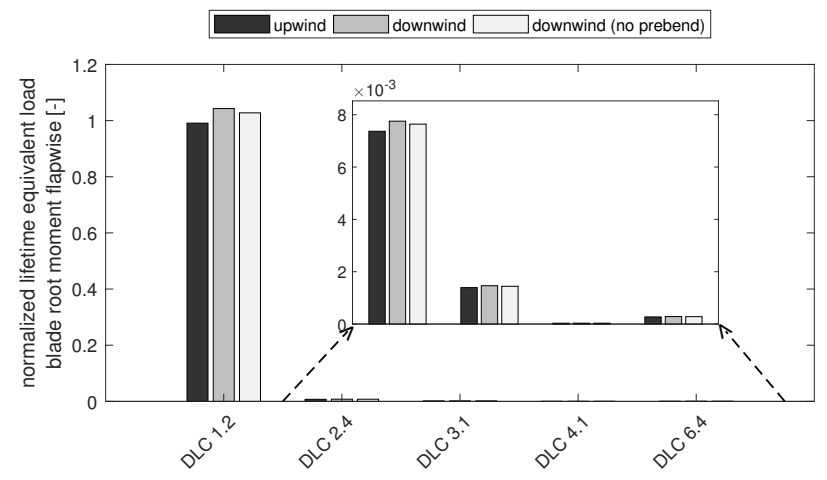

FIGURE 6 Comparison between the upwind configuration, the downwind configuration and the downwind configuration without prebend in normalized lifetime equivalent load for the flapwise blade root bending moment. Evaluated design load cases are: DLC 1.2 normal operation, DLC 2.4 power production with large yaw errors, DLC 3.1 Start-up in normal wind profile DLC 4.1 shut- down in normal wind profile, DLC 6.4 parked.

More than $99 \%$ of the lifetime equivalent load in all three configurations is determined by the normal operation (DLC 1.2). The figure shows that the normalized lifetime equivalent load for blade root flapwise bending is in the downwind configuration $5 \%$ higher and in the downwind configuration without prebend $4 \%$ higher than in the upwind configuration.

To quantify the effect of the tower shadow on the fatigue load, DLC 1.2 has been simulated in the two downwind configurations with the potential flow model. The tower shadow model exchange decreased the flapwise fatigue load in the blade root by $5 \%$ in the downwind configuration and by $4 \%$ in the downwind configuration without prebend. Thus, the same blade root flapwise fatigue load level as in the upwind configuration is achieved and the tower shadow is seen to be the causation of the increase in flapwise blade root fatigue loads.

Table 2 shows the lifetime equivalent load of the investigated sensors for normal operation.

The table shows a general increase of the fatigue loads when the upwind configuration is changed into a downwind configuration. The only exceptions are fatigue loads of the longitudinal tower bottom bending moment and the main bearing yaw moment, which are decreasing in the downwind configurations, compared to the upwind configuration. The fatigue load of tower bottom flange torsion remains unchanged for the downwind configuration, and decreases slightly in the downwind configuration without prebend, compared to the upwind configuration. Removing the prebend in the downwind configuration changes the relative difference in fatigue load level compared to the upwind configuration for most sensors not more than $2 \%$. Two exceptions for the downwind configuration without prebend are the blade root torsion which increases from $2 \%$ to $45 \%$ above the fatigue load level of the upwind configuration and the blade root edgewise fatigue load which increases from $7 \%$ to $11 \%$ higher fatigue load than in the upwind configuration.

With the exchange of the tower shadow model from the jet-flow deficit model to the potential flow model for the downwind configuration, the fatigue load of the tower lateral bending and the blade root flapwise bending are seen to reach the same level as in the upwind configuration. For the downwind configuration with potential flow model the tower torsion and the main bearing tilt fatigue load decrease to a fatigue load level $2 \%$ below the level of the upwind configuration. For the downwind configuration with potential flow model the main bearing yaw fatigue load level is seen to decrease to a load level $3 \%$ below the load level of the upwind configuration. For the tower longitudinal bending the fatigue load level decreases to a fatigue load level $4 \%$ below the level of the upwind configuration.The blade torsion fatigue load of the downwind configuration decreases $14 \%$ below the level of the upwind configuration with the change to a potential flow model. In case of the edgewise blade root bending a fatigue load reduction of $2 \%$ is observed with the exchange of the tower shadow model from jet-flow deficit to potential flow for the downwind configuration. However, the fatigue load does not reduce to the level of the upwind configuration. For the tower lateral fatigue load no load reduction can be observed for the downwind configuration with the exchange of the tower shadow model to the potential flow model.

The tower shadow is seen to be the main reason for the increase in fatigue loads when the upwind configuration is changed into a downwind configuration. The reduction of the fatigue load level for the downwind configurations and the downwind configuration with potential flow to a level below the fatigue load of the upwind configuration can be explained with less variation of the inflow condition. In the downwind configuration, the tilt angle counter acts the inclination angle of the wind. In the upwind configuration on the other hand, the tilt and the inclination angle both lead to an increased flow variation over the rotor plane. The blade torsion fatigue load increases drastically when the prebend is removed from the downwind configuration due to the gravity loading when the blade deflects further out of the rotor plane. The lower damping of the first edgewise forward whirling mode explains the increase of fatigue loads for the edgewise blade root moment in the downwind configurations, which is not due to the tower shadow. The tower lateral bending is coupled to the edgewise motion of the blades and excited by the lower damped blade motion in 



FIGURE 7 Comparison of the upwind configuration, the downwind configuration and the downwind configuration without prebend. On the Left: comparion of normalized power curve from DLC 1.2 without yaw misalignment, plotted with bin averages for $1 \mathrm{~m} / \mathrm{s}$ bins. On the right: comparison of the normalized difference in power power production between downwind configurations and upwind configuration.

the downwind configuration. The increase in the tower lateral fatigue load when changing an upwind into a downwind configuration is not due to the tower shadow.

\section{4 | Power output}

In the following section, the power production of the three turbine configurations the annual energy production as well as the power fluctuations are discussed. Figure 7 hows the normalized power curves and the difference in power over wind speed.

The figure shows on the left that the power production in all three configurations, upwind and downwind and downwind without prebend, is similar below rated power. It can be seen that the upwind configuration has a slightly higher power output than the two downwind configurations below rated power. The downwind configuration without prebend shows the lowest power production of the three configurations, below rated power. Above rated power no difference between the configurations is observed. The maximum difference is a $1 \%$ lower power production at $8 \mathrm{~m} / \mathrm{s}$ in the downwind configuration than in the upwind configuration. For the downwind configuration without prebend it can be seen that the losses are significantly higher, up to $2.3 \%$ at $8 \mathrm{~m} / \mathrm{s}$, compared to the upwind configuration. Overall, this leads to a $0.75 \%$ lower AEP in the downwind configuration and a $1.66 \%$ lower AEP in the downwind configuration without prebend, compared to the upwind configuration. With the exchange of the jet-flow deficit model to the the potential flow tower shadow model the downwind configuration showed an AEP gain of $0.77 \%$ compared to the upwind configuration. Exchanging the tower shadow model to the potential flow model for the downwind configuration without prebend gives an AEP loss of $0.1 \%$ compared to the upwind configuration. Simulating the power curves without inclination angle leads to a $1.97 \%$ lower AEP in the downwind configuration than in the upwind configuration. Without the inclination angle the downwind configuration without prebend shows $2.78 \%$ less $A E P$ than the upwind configuration.

Without the losses from the tower shadow jet-flow deficit model the downwind configuration would show a higher power production since the tilt angle aligns the rotor plane better with the inclination angle of the inflow. The alignment of inclination and tilt angle overcomes losses due to a decreased rotor area, as the cone is in the same direction as the blades deflect under loading for the downwind configurations (see Figure 1 . Without the prebend in the downwind configuration, the decrease in the rotor area is too large and even with the potential flow tower shadow model the AEP production is lower than in the upwind configuration. Excluding the influence of the inclination angle therefore increases the difference in power production, as the decrease in rotor area decrease becomes the dominant effect.

Figure 8 shows the normalized power fluctuations over the wind speed for the simulations without yaw misalignment or inclination angle. The figure shows that the power fluctuations increase for wind speed bins around rated power up to $5 \%$ when the downwind configuration is compared to the upwind configuration for equal inflow conditions on the rotor. For the wind speed bins at $5 \mathrm{~m} / \mathrm{s}$ and $6 \mathrm{~m} / \mathrm{s}$ and wind speed bins above $13 \mathrm{~m} / \mathrm{s}$ the fluctuations are decreased up to $3 \%$ below the level of the upwind configuration. For the downwind configuration without prebend the fluctuation is increased up to $13 \%$ for the wind speed bin of $11 \mathrm{~m} / \mathrm{s}$. The maximum decrease in fluctuations for the downwind configuration without prebend is up to $1 \%$ below the fluctuation level of the upwind configuration. Including the inclination angle decreases the fluctuation level of the downwind configurations. The maximum power fluctuation drops in the downwind configuration to $1 \%$ above the upwind fluctuation level. In all other wind bins the downwind configuration shows a lower fluctuation level than the upwind configuration. In the downwind configuration without prebend the maximum power fluctuation is $5 \%$ higher than the power fluctuation in the upwind configuration. Except for the two lowest wind speed bins the power fluctuation in the downwind configuration without prebend remains higher than in the upwind configuration. Exchanging the tower shadow model for the downwind configurations to the potential flow model decreases the power fluctuations for both downwind configurations. For nearly 


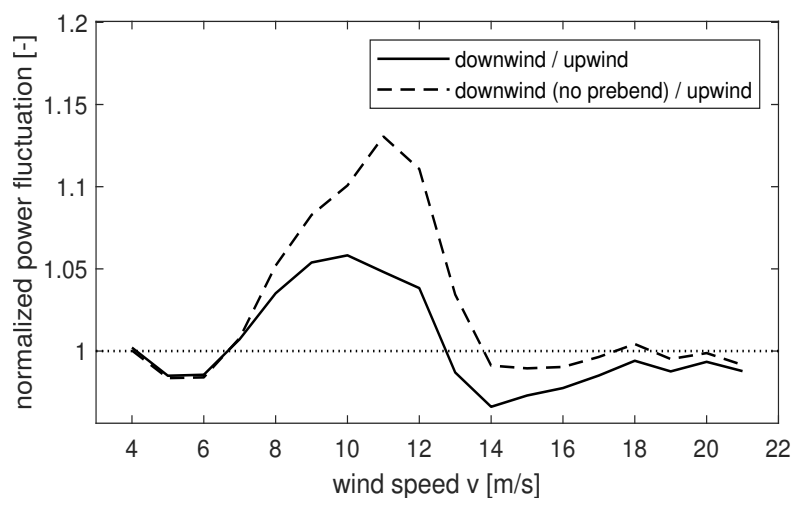

FIGURE 8 Normalized power fluctuation of the downwind and the downwind configuration without prebend from DLC 1.2 without yaw misalignment or inclination angle, plotted with bin averages for $1 \mathrm{~m} / \mathrm{s}$ bins normalized with the respective value of the upwind configuration.

all wind speed bins a power fluctuation level of the upwind configuration or lower is achieved for the downwind configuration. The power fluctuation level of the downwind configuration without prebend remains higher than for the downwind configuration and also remains higher than for the upwind configuration level around rated power.

Without inclination angle the differences in power fluctuations between the downwind configurations and the upwind configuration are determined by the lower edgewise damping of the downwind configurations and the tower shadow effect. Due to the lower edgewise damping the downwind configuration shows a higher power fluctuation level than the upwind configuration. The downwind configuration without prebend shows generally a higher power fluctuation level than the downwind configuration as the edgewise damping is the lowest. If the inclination angle is included the inflow conditions, the downwind configurations are subject to a inflow on the rotor plane with less variation than the upwind configuration. As the inflow in the downwind configurations is closer to being perpendicular to the rotor plane, it compensates for some of the tower shadow effect and some of the effect of the lower edgewise damping.

\section{4 | CONCLUSIONS}

The S111 2.1MW turbine in the upwind configuration has been compared to downwind configurations, with and without prebend. With the change into a downwind configuration an increase in tower clearance could be achieved. The initial unloaded tower clearance due to the prebend (62\% of the unloaded tower clearance of the upwind configuration) could be compensated by a $44 \%$ lower blade deflection in the downwind configuration, resulting into a $6 \%$ higher tower clearance. With a configuration without blade prebend, the minimum tower clearance could be increased by additional $11 \%$.

The downwind configurations are benefiting in the flapwise blade root moment from the fact that the coning direction is the same as the deflection direction of the blades in the critical situations. The flapwise extreme load is seen to be $10 \%$ lower in the downwind configurations.

The tilt angle is beneficial for the downwind configurations, as an inclination angle of the wind field is simulated. With the tilt angle a better alignment of the rotor plane with the inflow is achieved for the downwind configurations. The lower inflow variations are beneficial for fatigue loads, and a decrease in the yaw related loads, such as tower torsion or main bearing yaw load, as well as the fatigue load for the tower longitudinal bending is observed.

The tower shadow effect is seen to increase the flapwise blade root fatigue loads by $5 \%$ for the downwind configuration and by $4 \%$ for the downwind configuration without prebend. The flapwise fatigue load increase agrees with predictions in literature by for example Zahle et al. ${ }^{7}$ or Glasgow et al. .8 .

A lower edgewise damping is observed when the upwind configuration is changed into a downwind configuration. With the reduced edgewise damping the edgewise extreme loads are increased by $5 \%$ for the downwind configuration and $8 \%$ of the downwind configuration without prebend. The lower edgewise damping also contirbutes to the edgewise blade root fatigue load increase and a total increase of $7 \%$ for the downwind configuration and $11 \%$ for the downwind configuration without prebend is observed.

As observed also by Ning and Petch ${ }^{4}$, the gravity moment of rotor-nacelle assembly increases the bending loads in the tower bottom flange. Due to the gravity moment of the rotor-nacelle assembly an increase in the extreme tower bottom bending moment of $14 \%$ for the downwind configuration and $15 \%$ for the downwind configuration without prebend is observed, compared to the upwind configuration.

The interaction of several effects result into a small AEP loss of $0.75 \%$ in the downwind configuration. As the blades deflect in the coning direction 
under loading the rotor area in the downwind configurations is smaller than in the upwind configuration. The tower shadow effect also contributes to the AEP loss. The tilt angle on the other hand compensates for some of the losses as the rotor is closer to be perpendicular aligned with the wind direction in the downwind configurations than in the upwind configuration. The AEP loss increases to $1.66 \%$ if the prebend of the blade is neglected. A general increase in AEP due to the alignment of tilt and inclination angle, as observed by Yoshida et al ${ }^{3}$, could not be confirmed, since the inclination angles are not as large as observed by Yoshida et al..

Power fluctuations are seen to be influenced by the interaction of the tilt, the tower shadow effect and the lower edgewise damping. While the tilt angle is beneficially reducing the inflow variations the tower shadow increases the inflow variation. The lower edgewise damping increases power fluctuations further and as as result the power fluctuations of the downwind configurations are higher than the fluctuations of the upwind configurations.

\section{5 | DISCUSSION}

In this study a downwind configuration is assembled from the S1112.1MW turbine to see potential benefits in a configuration change from a loadand AEP-perspective. It has been seen that the downwind configuration in the current simulation set-up benefits from the tilt angle that counteracts the inclination angle. Since the IEC-standard is a guideline for the design of upwind configurations, the inclination angle is a conservative property of the inflow conditions. Thus, using the same inclination angle for the downwind configuration is not a fair comparison of the configurations. When comparing the concepts, the angle should either be inverted for the downwind configuration or both configurations should be subject to a flow field without inclination angle. The change in the inclination angle consequentially leads to a higher power difference between the configurations as well as an increase in fatigue loads in the downwind configuration and a decrease in fatigue loads in the upwind configuration.

The observed increase in blade fatigue loads for the downwind configurations means that the tower shadow model is crucial for the evaluation of the downwind configuration. The used model considers the deficit of the free wind speed behind the tower, but the increased turbulence is not modelled. More investigations need to be done to evaluate if this effect is relevant for the load fluctuations. A tower shadow validation will be needed to evaluate different tower shadow alleviation techniques, which would be required to reduce the loads to the level of the upwind configuration.

A significant increase in edgewise extreme and fatigue blade loads is observed due to a lower edgewise damping in the downwind configurations. It cannot yet be concluded if the lower edgewise damping is a general property of downwind configurations. Future work has to investigate the difference in the modal dynamics between upwind and downwind configurations, as this determines the dynamic stability of the turbine and is a relevant cause for differences in loads. A better understanding of the edgewise damping is needed to be able increase the damping if possible, to avoid high loads and instabilities.

The choice of the controller significantly influences the loads due to load cases where certain situations are handled. This means that loads could differ significantly with different handling routines of for example, turbine shut down or operation at high yaw errors. In this case extreme tower bending or flapwise blade root moment could be reduced in all three configurations with for example the proprietary controller. If this would change the difference between the upwind and the downwind configuration is unclear. Situation determined by normal operation including turbulence would not be improved. Thus, no improvements can be expected for the tower clearance or the edgewise loads. Also AEP losses would not be compensated with the proprietary controller since they are related to operation below rated power.

Seen from the perspective of blade design, it could be possible to reduce blade material and blade cost for the chosen geometry assembly when changing the upwind into a downwind configuration. Firstly, because the tower clearance is increased, secondly because the extreme flapwise blade load is decreased significantly. These two advantages might overcome the increase in extreme edgewise blade loads.

However, from the full turbine perspective it is doubted that a pure material reduction of the blades is beneficial. To exploit the full potential of the downwind configuration, the LCOE should be considered. The LCOE can be expected to increase with the configuration change, since the tower is a major cost component and the load has significantly increased when the upwind configuration is changed into a downwind configuration. Further, the downwind configurations showed a lower AEP, increasing the LCOE compared to the upwind configuration. Consequentially, if a cost competitive downwind turbine should be designed, the blade moulds could not be kept. Instead a new rotor should be designed while the load envelope is kept the same as in the upwind configuration.

With this approach the rotor diameter could be increased to harvest more energy. The increase of rotor diameter would be limited by the flapwise blade root moment. If tower clearance becomes a design constraint for a larger rotor diameter, the cut-out wind speed could be reconsidered, since the the contribution to the AEP is relatively low. To avoid a significant increase in the tower cost the static loading the downwind configuration should be modified, such that the center of gravity of the rotor-nacelle assembly is located closer to the tower center. In that case, the gravity moment would decrease. Since the static moment of the downwind configurations can never have a contribution reducing the extreme tower bottom bending moment, as it does in the upwind configuration, the thrust level for the downwind configuration would have to be reduced significantly. In that case the tower cost could potentially reach a tower cost level of the upwind configuration. From a limited thrust level a further reduction 
in the extreme flapwise bending moment could be expected, potentially allowing further rotor diameter increase. In order to fairly evaluate if such drastic changes in design strategy are beneficial for a downwind configuration, the upwind configuration would also need to be redesigned. A limited thrust level would be expected to be also beneficial for an upwind configuration. Only in that case a fair comparison could be made and it could be evaluated if the LCOE of a downwind configuration is lower than the LCOE of an upwind configuration.

Overall it can be concluded that there is no clear benefit of changing an existing upwind configuration into a downwind configuration. To see the benefits a redesign of both configurations would be required to evaluate the benefit in LCOE.

\section{Funding Information}

This Project is an industrial PhD project founded by the Innovation Fund Denmark (case number 5189-00180B) and Suzlons Blade Science Center.

\section{References}

1. Kiyoki S, Sakamoto K, Inamura S, Tobinaga I, Saeki M, Yokoyama K. Development of 5-MW Downwind Turbine and Floating Substation Facility for Offshore Wind Power. Hitatichi Review. 2016;65(4):938-943. http://www.hitachi.com/rev/pdf/2016/r2016_04_all.pdf. Accessed: April 26, 2018.

2. Kress C, Chokani N, Abhari RS, et al. Impact of flow inclination on downwind turbine loads and power. Journal of Physics: Conference Series 753 : The Science of Making Troque from Wind (TORQUE2016). 2016;. doi: 10.1088/1742-6596/753/2/022011.

3. Yoshida S. Performance of Downwind Turbines in Complex Terrains. Wind Engineering. 2006;30(6):487-502.

4. Ning A, Petch D. Integrated design of downwind land-based wind turbines using analytic gradients. Wind Energy. 2016;19:2137-2152. doi: 10.1002/we.1972.

5. Glasgow JC, Corrigan RD. Results of Free Yaw Test of the Mod-0 100-Kilowatt Wind Turbine. NASA Report. 1983;TM-83432:1-16.

6. Corrigan RD, Viterna LA. free yaw performance of the MOD-0 large horizontal axis 100kW wind turbine. NASA-Report. 1982;TM83(19235):103-122.

7. Zahle F, Madsen HAa, Sørensen NN. Evaluation of tower shadow effects on various wind turbine concepts. Research in Aeroelasticity DTU Report EFP-2007-II. 2009;1698:1-147.

8. Glasgow JC, Miller DR, Corrigan RD. Comparison of upwind and downwind rotor operations of the DOE/NASA IOO-kW MOD-0 wind turbine. NASA Report. 1981;TM-8744:225-234.

9. Reiso M, Muskulus M. The simultaneous effect of a fairing tower and increased blade flexibility on a downwind mounted rotor. Journal of Renewable and Sustainable Energy. 2013;5(3):033106-1-1033106-11. doi: 10.1063/1.4803749.

10. IEC . IEC 61400-1 ed.3 Wind turbines- Part1: Design requirements. : International Electronical Commission; 2014.

11. Larsen TJ, Hansen AM. How 2 HAWC2, the user's manual. Risø-Report:Risø-Report. 2014;R1597(verion 4-5):1-147.

12. Hansen MH, Thomsen K, Natarajan A, Barlas A. Design Load Basis for onshore turbines Revision 00. DTU Report; DTU Wind Energy. 2015;E-0074.

13. Madsen H. Aa., Riziotis V., Zahle F., et al. Blade element momentum modelling of inflow with shear in comparison with advanced model results. Wind Energy; Vol. 15; p. 63-81. 2011;.

14. Hansen MH, Henriksen LC. Basic DTU Wind Energy Controller. DTU-Wind-Energy-Report. 2013;E-0028.

15. Hansen MH. Aeroelastic Stability Analysis of Wind Turbines Using an Eigenvalue Approach. Wind Energy. 2004;7:113-143. doi: 10.1002/we.116. 
TABLE 1 Difference in the highest normalized extreme value between the upwind and the downwind configuration, as well as the upwind and the downwind configuration without prebend, and the design driving load cases for all three configurations. Design driving load cases are: DLC 4.2 shut down during extreme operational gust, DLC 6.2 idling at abnormal yaw error, DLC 1.3 power production in extreme turbulence, DLC 2.2y operation at extreme yaw errors.

\begin{tabular}{|c|c|c|c|c|c|c|}
\hline \multirow[t]{2}{*}{ load sensor } & \multicolumn{2}{|c|}{$\Delta$ normalized extreme load [-] } & \multicolumn{3}{|c|}{ DLC } & \\
\hline & downwind & $\begin{array}{l}\text { downwind } \\
\text { (no prebend) }\end{array}$ & upwind & downwind & $\begin{array}{l}\text { downwind } \\
\text { (no prebend) }\end{array}$ & \\
\hline tower bottom flange longitudinal & +0.14 & +0.15 & DLC 4.2 & DLC 4.2 & DLC4.2 & Figure $\sqrt{5}$ \\
\hline tower bottom flange lateral & +0.10 & +0.08 & DLC 6.2 & DLC 6.2 & DLC 6.2 & \\
\hline tower bottom flange torsional & -0.06 & -0.09 & DLC 1.3 & DLC 1.3 & DLC 1.3 & \\
\hline blade root flange flapwise & -0.10 & -0.10 & DLC 2.2y & DLC 2.2y & DLC 2.2y & Figure 3 \\
\hline blade root flange edgewise & +0.05 & +0.08 & DLC 1.3 & DLC 1.3 & DLC 1.3 & \\
\hline blade root flange torsional & +0.05 & +0.03 & DLC 6.2 & DLC 6.2 & DLC 6.2 & \\
\hline main bearing tilt & +0.02 & +0.05 & DLC $2.2 y$ & DLC 1.3 & DLC 1.3 & \\
\hline main bearing yaw & -0.07 & -0.10 & DLC 1.3 & DLC 1.3 & DLC 1.3 & \\
\hline
\end{tabular}


TABLE 2 Difference in the normalized lifetime equivalent load between the upwind configuration, the downwind configuration and the downwind configuration without prebend for DLC 1.2 (normal operation).

\begin{tabular}{|c|c|c|c|}
\hline \multirow[t]{2}{*}{ load sensor } & \multicolumn{2}{|c|}{$\Delta$ normalized life time equivalent load [-] } & \\
\hline & downwind vs. upwind & downwind (no prebend) vs. upwind & \\
\hline tower bottom flange longitudinal & -0.02 & -0.03 & \\
\hline tower bottom flange lateral & +0.03 & +0.05 & \\
\hline tower bottom flange torsion & -0.00 & -0.01 & \\
\hline blade root flange flapwise & +0.05 & +0.04 & Figure 6 \\
\hline blade root flange edgewise & +0.07 & +0.11 & \\
\hline blade root flange torsion & +0.02 & +0.45 & \\
\hline main bearing tilt & +0.06 & +0.04 & \\
\hline main bearing yaw & -0.02 & -0.04 & \\
\hline
\end{tabular}


How to cite this article: G. Wanke, L. Bergami, T. J. Larsen, and M. H. Hansen, (20XX), Changes in design driving load cases; operating an upwind turbine with a downwind rotor configuration., Wind Energy, 20XX;XXXX. 


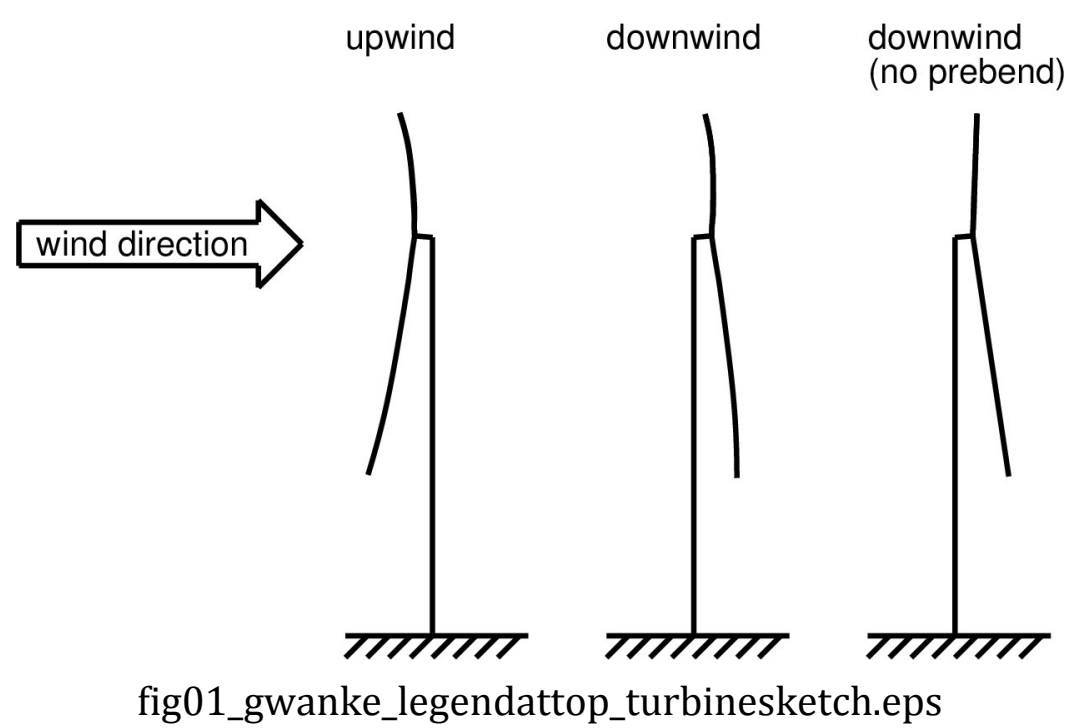

This article is protected by copyright. All rights reserved. 


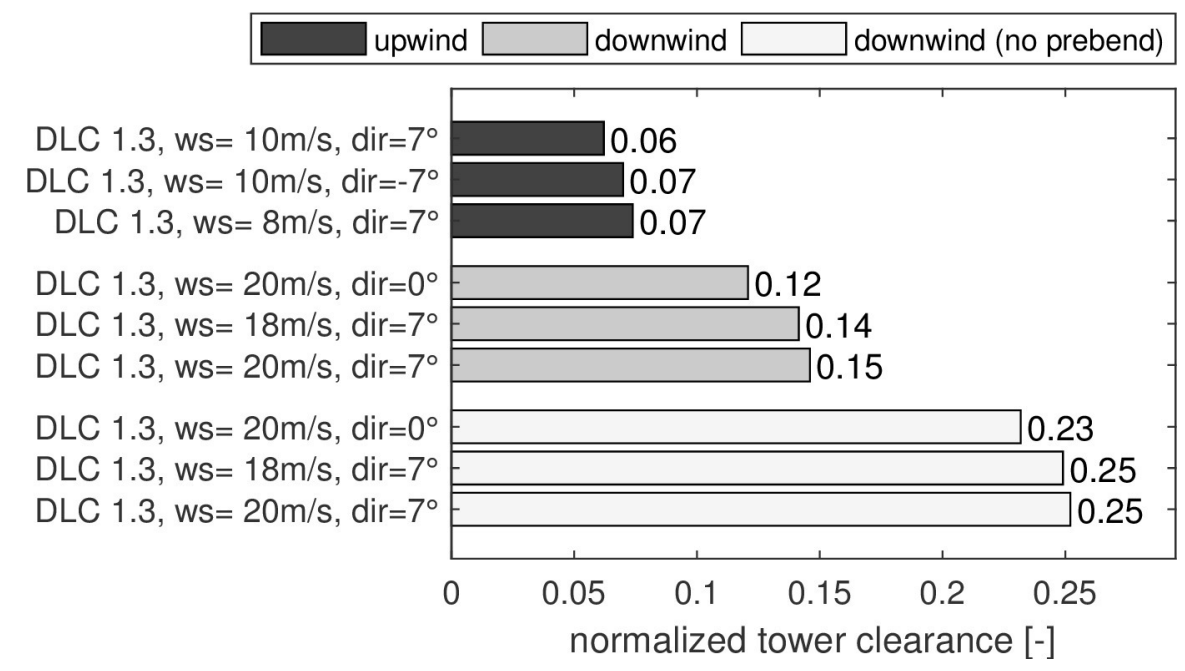

fig02_gwanke_legendattop_towclear.eps 

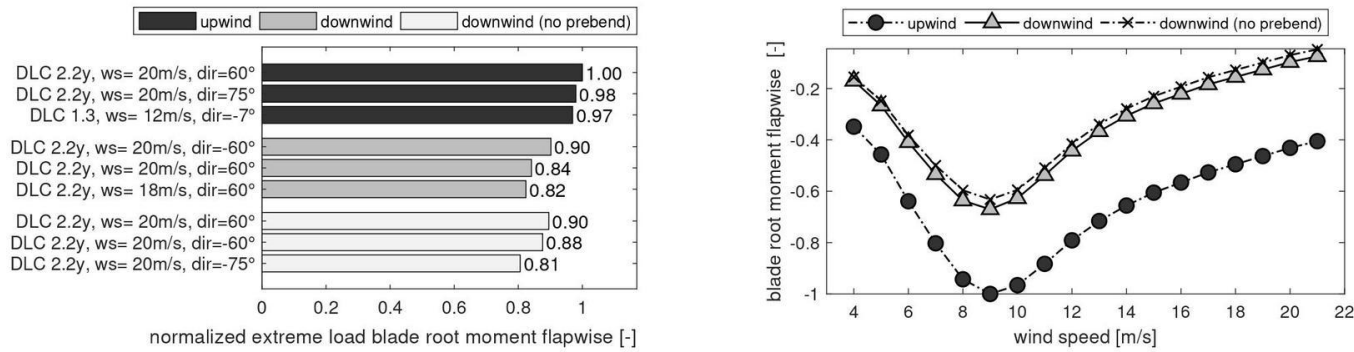

fig03_GWanke_LegendAtTop_Blade_flapwise.jpg

This article is protected by copyright. All rights reserved. 

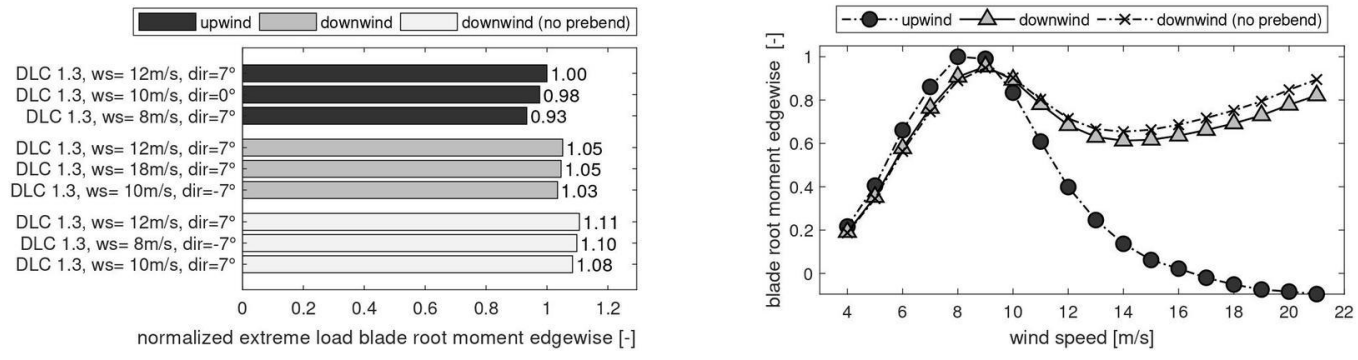

fig04_GWanke_LegendAtTop_Blade_edgewise.jpg

This article is protected by copyright. All rights reserved. 

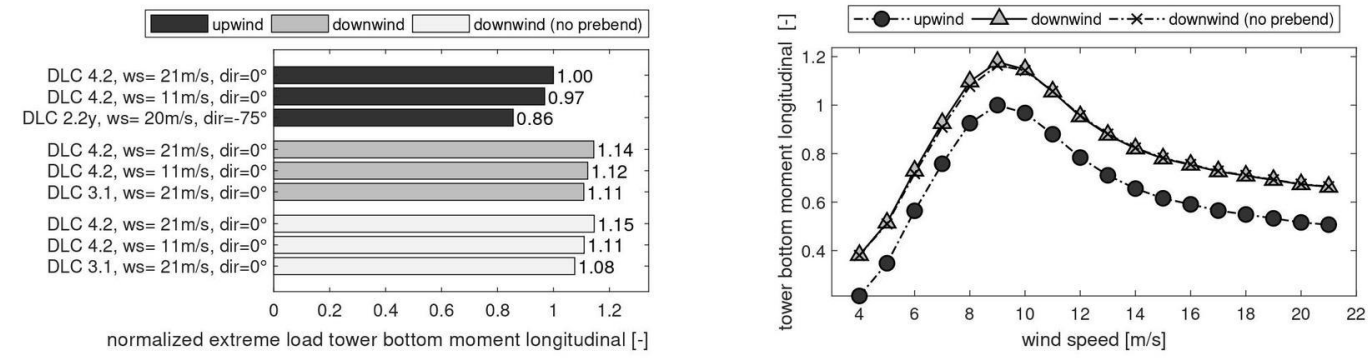

fig05_GWanke_LegendAtTop_tower_longitudinal.jpg

This article is protected by copyright. All rights reserved. 


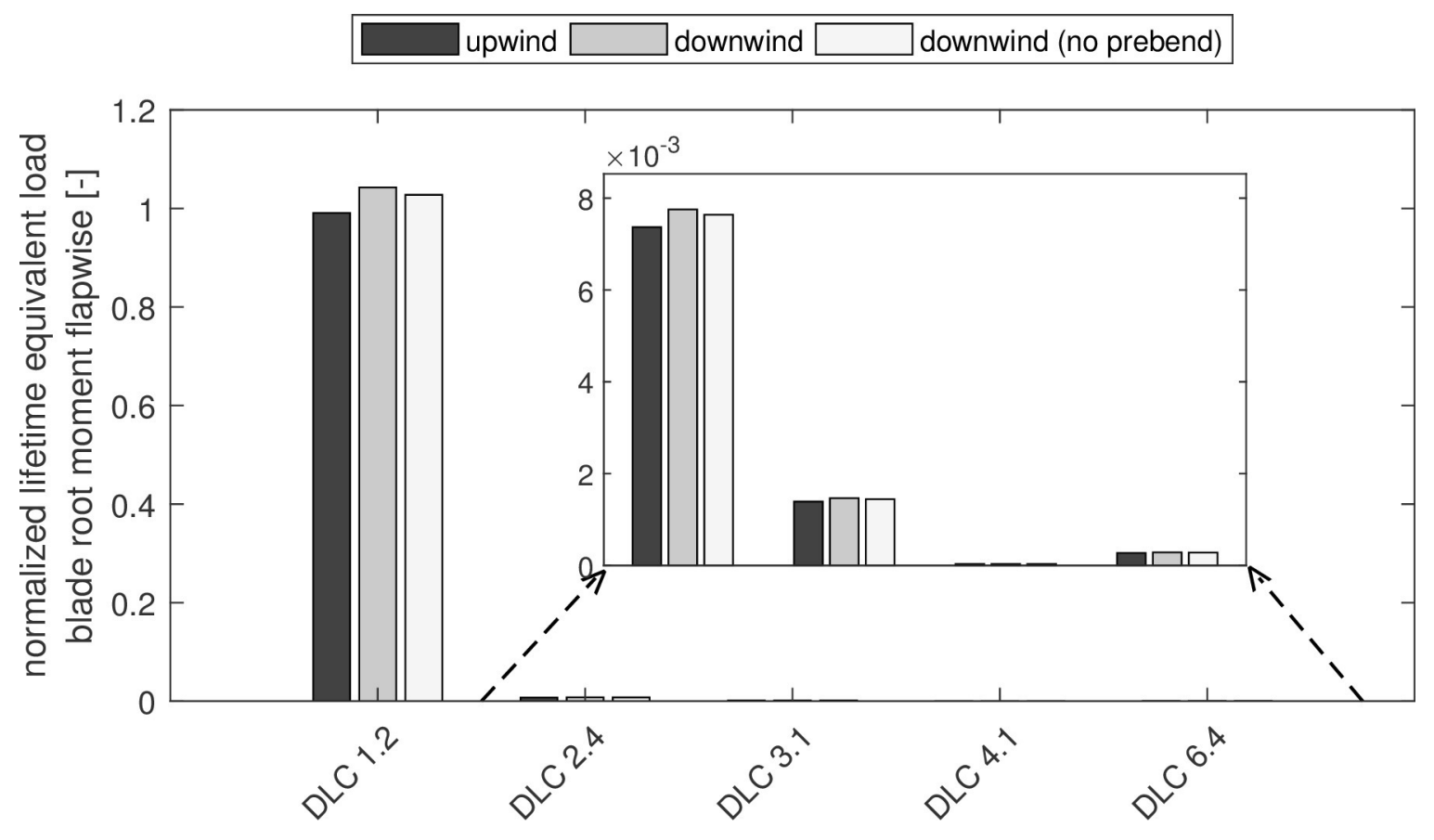

fig06_gwanke_legendattop_lifetimeeqload_flapwise.eps

This article is protected by copyright. All rights reserved. 

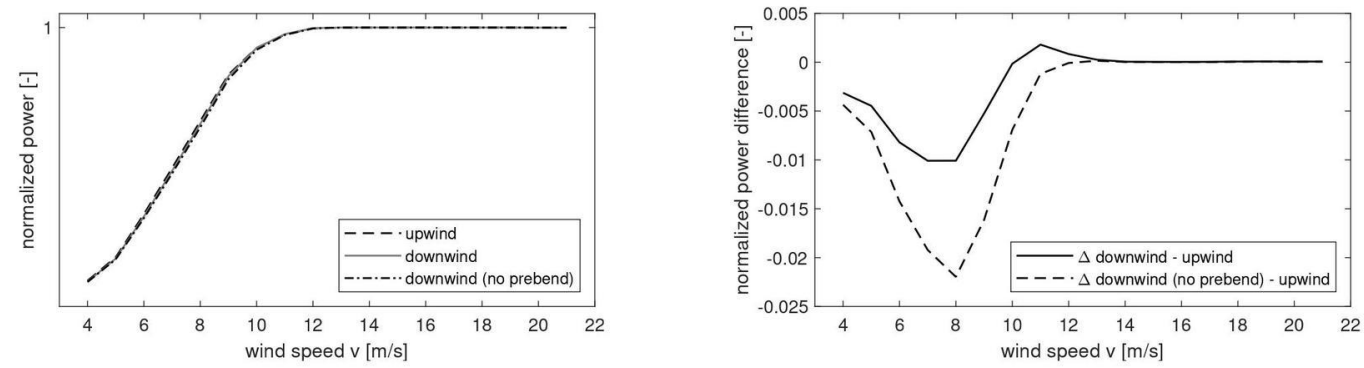

fig07_GWanke_LegendAtBottom_Powercurve.jpg

This article is protected by copyright. All rights reserved. 




fig08_gwanke_legendattop_powerfluctuation.eps

This article is protected by copyright. All rights reserved. 